To be published in IEEE

BNL非 22465

Trans. Nucl. Sci., June 1977

Invited paper presented at the 1977 Particle Accelerator Conference, 16-18 March, 1977, Chicago, Illinois

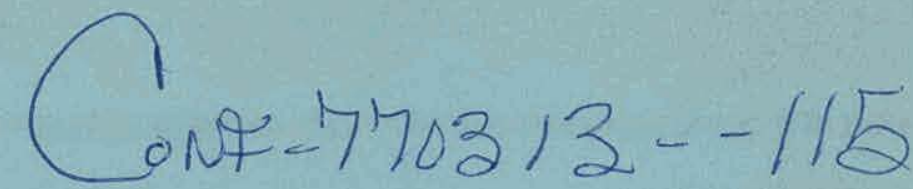

\title{
COMPUTER NETWORKS IN FUTURE ACCELERATOR CONTROL SYSTEMS*
}

D. Gerd Dimmler

Brookhaven National Laboratory

Upton, New York 11973

March 1977

* Research carried out under the auspices of the Energy Research and Development Administration: Contract No. EY-76-C-02-0016. 


\section{DISCLAIMER}

This report was prepared as an account of work sponsored by an agency of the United States Government. Neither the United States Government nor any agency Thereof, nor any of their employees, makes any warranty, express or implied, or assumes any legal liability or responsibility for the accuracy, completeness, or usefulness of any information, apparatus, product, or process disclosed, or represents that its use would not infringe privately owned rights. Reference herein to any specific commercial product, process, or service by trade name, trademark, manufacturer, or otherwise does not necessarily constitute or imply its endorsement, recommendation, or favoring by the United States Government or any agency thereof. The views and opinions of authors expressed herein do not necessarily state or reflect those of the United States Government or any agency thereof. 


\section{DISCLAIMER}

Portions of this document may be illegible in electronic image products. Images are produced from the best available original document. 
COMPUTER NETWORKS IN FUTURE ACCELERATOR CONTROL SYSTEMS*

\section{Gerd Dimmler}

Brookhaven National Laboratory Upton, New York 11973

\section{$\underline{\text { Summary }}$}

Some findings of a study concerning a computer. based control and monitoring system for the proposed ISABELLE Intersecting Storage Accelerator are presented. Requirements for development and implementation of such a system are discussed. An architecture is proposed where the system components are partitioned along functional 1ines. Implementation of some conceptually significant components is reviewed.

\section{Background}

The cost of electronic computer equipment has decreased over the past several years to a point where it has become a well accepted concept to use many computers interconnected as a network within control systems of the complexity necessary in accelerators, rather than one central computer. Several recently developed and. proposed systems emphasize this view ${ }^{1-4}$.

The Brookhaven National Laboratory is presently in the process of proposing construction of an Intersecting Storage Accelerator, ISABELLE 5 , in the following referred to as ISA. Such an undertaking requires an effort to study and to define appropriate architectures of a control system for such an accelerator on a fundamental level. Some initial findings of a study concerning a possible ISA control and monitoring system are presented.

It is a significant constraint in the development of a complex control system that detalled specifications for the system can only be determined after the parameters of the machine to be controlled are known; yet the system mist be completed in many of its functions before the machine to be controlled is operational. The latter portion of this statement takes into consideration that

(1) a complex accelerator, such as the ISA, requires a major portion of the control system for initial tests and initial operations; and

(2) modifications, as contrasted to expansions, of inftially established control philosophies and functional architectures, as well as of fundamental hardware and software structures would likely lead to unacceptable delays in the completion of such a control system and of the accelerator. Architectural and structural modifications, thus, should be avoided.

The situation is complicated by the fact that such a control system interacts with almost every part of the accelerator to be controlled; control system designers have to interact closely with the various accelerator development and construction groups. There are, thus, severe time and scheduling constraints. For useful interaction with accelerator designers, the fundamental architecture of the control system must be well determined at an early stage of construction. Then the numerous control functions to be implemented within the architecture can be determined as construction progresses. At. this early phase the study concentrates on architectural and structural considerations.

\section{Partitioning}

It is a unique property of computer systems in general, not necessarily existing in other complex systems, that the two major implementation structures, the hardware and the software, developed quite independently over the past three decades, although they are very closely correlated to one another. This situation did not lead to immediate difficulties in the case of the large central computer systems of the past decade. There, the properties of the selected central processor, such as a PDP10, a SIGMA series processor, etc., served as a focal point for software development personnel, hardware interface engineers and for support personnel involved in the project. Not infrequently the particular properties of such a processor served as a basis for a new branch of computer systems technology in the field of software engineering 6 as well as in the field of hardware interfacing.

The process of partitioning of a system into defined components was well established within hardware structures for reasons of economy and maintainability. In software engineering this process was often considered a detriment to flexibility. Difficulties arose in those systems due to the lack of comprehensive software structures which sometimes led to systems with undesireable behavior. Another important property of these central systems was that limitations on the number and the types of developments considered reasonable served as an implicit limitation on the size of hardware and software support staff and, thus, served as an implicit operating cost control.

The situation is very different in the case of a network, where a computer processor is just one of many hardware components. Many processors, possibly of different types, may exist in the control system. If used as a focal point the different properties of the processors may lead to confusion ${ }^{8}$, disintegration and to possible failure of the system design and the subsequent system support. A new strategy for the design of such a system and a new level of system description ${ }^{7}$ seem to be indicated.

A fundamental question to be solved in the design of such a control system is: how to partition the services to be performed by the system into the various elements of the network. An element is meant to be here a hardware/software combination. It is evident that the partitioning process must take place on the functional level, a matter of considerable significance here.

A strategy of system design is used as a basis which takes the above observations into consideration. It leads to a network architecture called functional distribution. The strategy is described in detail elsewhere ${ }^{9}$. In particular, as indicated at an example in Fig. 1, the sequence of steps to be taken is:

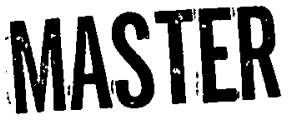

* This work was carried out under the asgpices of the Energy Research and Development Administration: Contract No. EY-76-C-02-0016. Research and Development Administration, nor any ors
of their contrators. 


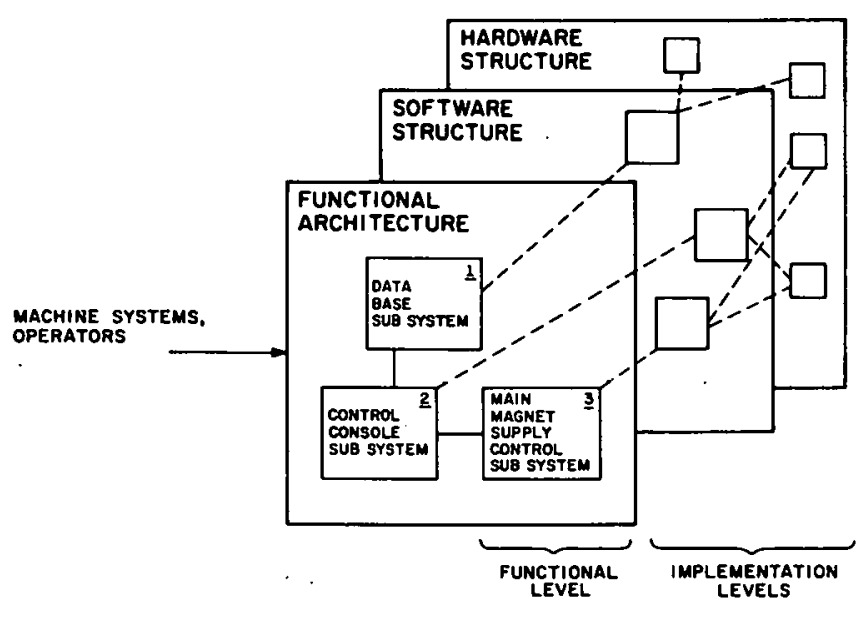

Figure 1

Levels of System Description and Implementation.

(1) the entire system to be designed is studied at the functional level;

(2) the functions are partitioned into sets of hierarchically organized, functional subsystems which are interconnected at the functional level. The resulting network description is in the following referred to as the functional architecture.

The partitioning process must be carried out in such a manner that a functional complexity level is reached which contains a set of functional subsystems having at least the following two properties:

(a) The boundaries (i.e., the inputs and outputs) of each functional subsystem in the set have approximately the saue cumplexity level and approximately uniform structure; and

(b) the functional subsystems in the set must be separable into subsets which do not overlap each other with respect to the software structures and hardware structures which are required to implement the subfunctions.

When this functional level has been reached each isolated functional subsystem is confined to a functional node of the system. In the example of Fig. 1 the data base subsystem would constitute a functional node, while the control console subsystem and the main magnet supply subsystem would be members of the same node.

Afteir this is accomplished, the functional nodes are partitioned into their two implementation structures, the hardware structure and the software structure. Iterations of the above are necessary in order to meet other constraints and conditions such as:

(1) to find an architecture where, for reasons of economy and conservation of development and support cost, suitable existing implementation structures are applicable;
(2) to keep, for reasons of economy, rellability and maintainability, the number of different types of components in the functional architecture, as well as in the two implementation structures, at a practical minimúm; and

(3) to provide for the use, wherever possible, of components in the implementation levels and in the functional level which match with future trends of partitioning and component development in the computer industry and in the electronics industry.

Properties of Some System Design Parameters

The response time, which is here defined as the time interval between a service request and the resulting response, is probably the most significant and influential design parameter in real-time systems. In - order to gain insight into key syetem design parameters it appears to be reasonable to partition, initially, such a real-time system into sets of subsystems with similar allowable response times. Such a partitioning is performed in Fig. 2. A decalled description is given elsewhere ${ }^{9}$ of which the following is a summary.

It is reasonable to group the key system design parameters broadly on the basis of their impact to cost types because cumputer control systems always represent a major cost.itrm. The following cost types are here considered:

(1) investment cost during the initial development;

(2) operating cost after completion; and

(3) depreciation cost, where only the impact of technological obsolescence is here considered.

There is a direct relationship between the economically achievable response time within a system and the investment cost in resource sharing systems. The longer the allowable response time requirements, the more efficiently available resources can be managed. Elaboration is given elsewhere ${ }^{10}$.

The expected mean time between changes (MTBC) has significant impact on the operating cost of a subsystem. This is particularly evident for sets of functional subsystems which include:

(1) complex system software; and

(2) inflexible hardware interconnections.

This type of operating cost is, in part, generated by:

(1) the hardware development cost generated by the change;

(2) the software development cost generated by the change;

(3) " hardware and, particularly, software rendered obsolete; and

(4) the unsettling effects of new operating procedures and new design errors which are, as experience shows, often caused by software changes.

A second parameter influencing operating cost, the damage caused by unisolated malfunctions, has often been overlooked in the design of systems. For instance, a malfunction in a random-access memory is a relatively minor event which may cause disastrous effects. Unrelated functions in the system may be arbitrarily interrupted causing possible loss of context and files. This situation can occur if the consequences of the malfunction were not.confined to the subsystem where the malfunction occurred. It is 


\begin{tabular}{|c|c|c|c|c|c|c|}
\hline RELATED TO $\rightarrow$ & \multicolumn{2}{|c|}{$\begin{array}{l}\text { INVESTMENT } \\
\operatorname{COST}\end{array}$} & \multicolumn{2}{|c|}{$\begin{array}{l}\text { OPERATING } \\
\text { COST }\end{array}$} & \multirow{3}{*}{\begin{tabular}{|l|}
$\begin{array}{c}\text { DEPRECIATION } \\
\text { COST }\end{array}$ \\
TECHNICALLY \\
USEFUL LIFE \\
SPAN OF \\
DES IGNS
\end{tabular}} & \multirow{3}{*}{$\begin{array}{l}\text { Remarks } \\
\text { Reason for } \\
\text { sharing of digi- } \\
\text { tal resources }\end{array}$} \\
\hline $\begin{array}{l}\text { DES IGN PATAMETERS } \\
\text { PROPERTIES } \rightarrow\end{array}$ & \multirow{2}{*}{$\begin{array}{l}\text { ALLOWABLE } \\
\text { RESPONSE } \\
\text { TIME }\end{array}$} & \multirow{2}{*}{$\begin{array}{l}\text { APPLICABIL- } \\
\text { ITY OF } \\
\text { SUBSYSTEMS }\end{array}$} & \multirow{2}{*}{$\begin{array}{l}\text { MEAN TIME } \\
\text { BETWEEN } \\
\text { CHANGES } \\
\text { (MTBC) }\end{array}$} & \multirow{2}{*}{\begin{tabular}{l|} 
DAMAGE \\
TO NETWORK \\
CAUSED BY \\
UNISOLATED \\
MALFUNC- \\
TIONS \\
\end{tabular}} & & \\
\hline $\begin{array}{l}\text { SUBSYSTEM } \\
\text { SETS. } \downarrow\end{array}$ & & & & & & \\
\hline $\begin{array}{l}\text { PERIPHERAL } \\
\text { OUTPUT } \\
\text { SUBSYSTEMS }\end{array}$ & $>10^{1} \mathrm{sec}$ & \multirow{2}{*}{$\begin{array}{l}\text { Functional } \\
\text { Subsystems } \\
\text { Applicable }\end{array}$} & \multirow{2}{*}{ LONG } & \multirow{2}{*}{$\mathrm{HIGH}^{*}$} & \multirow{2}{*}{$\begin{aligned} & \text { LONG } \\
> & 5 \text { years }\end{aligned}$} & \multirow{2}{*}{$\begin{array}{l}\text { (1) Economic: } \\
\text { (a) High cost of } \\
\text { output devices; } \\
\text { (b) High cost of } \\
\text { storage devices. } \\
\text { (2) Logistic: } \\
\text { (a) sharing of data } \\
\text { and procedures; } \\
\text { (b) merging of file } \\
\text { contents. }\end{array}$} \\
\hline $\begin{array}{l}\text { FILE/DATA, } \\
\text { SUBSYSTEMS }\end{array}$ & $\sim 10^{-1} \mathrm{sec}$ & & & & & \\
\hline $\begin{array}{l}\text { CONTROL } \\
\text { APPLICATION } \\
\text { SUBSYSTEMS }\end{array}$ & $\sim 10^{-3} \mathrm{sec}$ & $\begin{array}{l}\text { Hardware \& } \\
\text { Syst. Softw. } \\
\text { generally } \\
\text { applicable }\end{array}$ & MEDIUM & MEDIUM & $\begin{array}{l}\text { MEDIUM } \\
\sim 3 \text { years }\end{array}$ & $\begin{array}{l}\text { Economic: } \\
\text { cost of } \\
\text { processors, } \\
\text { (RAM) memory and } \\
\text { interconnections. }\end{array}$ \\
\hline $\begin{array}{l}\text { SIGNAL } \\
\text { CONDITIONING } \\
\text { ELECTRONICS } \\
\text { SUBSYSTEMS }\end{array}$ & $\sim 10^{-6} \mathrm{sec}$ & \multirow[t]{2}{*}{$\begin{array}{l}\text { Subsystems } \\
\text { very specific } \\
\text { components } \\
\text { generally } \\
\text { applicable }\end{array}$} & \multirow[t]{2}{*}{ SHORT } & \multirow[t]{2}{*}{$\operatorname{LOW}^{* *}$} & \multirow[t]{2}{*}{$\begin{aligned} & \text { SHORT } \\
\sim & 1 \text { year }\end{aligned}$} & \multirow[t]{2}{*}{$\begin{array}{l}\text { Economic: } \\
\text { cost of } \\
\text { digital- } \\
\text { electronics }\end{array}$} \\
\hline $\begin{array}{l}\text { PROCESS DEVICES } \\
\text { SENSORS / } \\
\text { ACTUATORS }\end{array}$ & $<10^{-6} \mathrm{sec}$ & & & & & \\
\hline
\end{tabular}

Figure 2

System Design Parameter Properties

\begin{abstract}
interesting to note that this kind of propagation of malfunctions is still often accepted in computer systems or, at most, meets minor concern.

The design of a subsystem is obsolete if it is no longer economically or technologically advantageous to duplicate such a design. It is a significant observation that the technically useful life span of hardware and software designs turn out to be long for functional subsystems which have a long allowable response time and tend toward complex system software; such as data bases and peripheral output subsystems: On the other hand, rapid advances in electronics technology, particularly in microminiaturization, often result in a short useful life span for designs within subsystems where the allowable response times are short.
\end{abstract}

\section{Scope}

The ISA control and monitoring system will manage data and control flows as well as maintain data bases and procedures necessary to provide at least the following global functions :

\section{(1) OPERATION .}

The machine always operates in one of the following major phases:
SETUP of parameters, data and procedures;

FILL the two rings;

ACCELERATE the beams. This 1s probably the most demanding phase for the control system;

MAINTAIN the stored beams;

TERMINATE or ABORT (dump) the beam s.

In addition, there may be test phases to be defined later.

(2) MODIFICATION

Data, parameters and procedures may be modified at suitable operational phases within independently set limits.

(3) MONITORING

The machine behavior will be selectively monitored.

(4) MEASU REMENT

Physical parameters will be selectively measured within independently set limits. 


\section{(5) MACHINE PRÖTECTION}

The machine will be protected against damage caused by malfunctioning equipment. This is a fairly. elaborate function. It will be aided by independent hardware protection systems within the varlous machine components.

(6) ENVIRONMENTAL PROTECTION

Some functions concerning the protection of personnel against health hazards and the protection of the environment will be provided.

(7) Other Functions

An array of support functions is expected from the system, such as:

(a) bookkeeping of schedules, information, etc.;

(b) small scale computation;

(c) maintenance of historical information;

(d) procedure (program) development.

\section{Some System Requirements}

Some of the key requirements to the'ISA control and monitoring system are listed below.

(1) The interaction of an operator, a physicist or other personnel with the control system must be comprehensive, non-confusing and straightforward. This requirement Implies that the interaction with the system is based on the execution of procedures which can be conveniently expanded, linked and nested. Straightforward operator interaction requires a careful control room layout. An example of an excellent layout is the control room 11 of the SPS accelerator at CERN.

(2) Procedure development should be very convenient.

(3) Monitoring displays must be comprehensive.

(4) A complete record of the accelerator status should be maintalned in real-time in the network within a reasonable time resolution.

(5) The consoles in the main control room should allow for access to all control and monitoring functions in the network. Other possible control rooms will, therefore, provide for additional access to a subset of those functions.

(6) Flexibility mist be provided in the sense that incremental expansions, improvements, and replacements can be made with prevailing technology, not the technology avallable at the time of the first phase system implementation.

\section{Reliability}

A network of the size and the cost of the ISA control and monitoring system is expected to experience a certain number of hardware malfunctions caused by deteriorating or faulty components. It cannot be entirely ruled out that, in the initial phases, malfunctions will also occur due to system software and hardware design errors and due to operator errors. A separate class of malfunctions is caused by design errors in undebugged application procedures. It is an important objective that the network as a whole stays operational in the presence of failing parts of the network. It follows that the effect of a malfunction must stay confined as close to the point of origin as structurally and economically feasible.

The mechanisms for detection and description of malfunctions and the confinement of malfunction effects play an important role in such a network 12 . Figure 3 shows the sequence of significant events if a malfunction occurs in the presence of simultaneously executed network procedures. The time intervals between the various events have a sigaficant influence on the probability of detection, on the precision of description, on the probability of proliferation of a malfunction and on the timeliness of a response. A low detection probability would cause the network to operate erratically with little opportunity to plan improvements. A low precision of malfunction description leaves 11ttle opportunity for manual or automatis failure resoveries. A high probability of malfunction proliferation would cause the..system to operate erratically.

\section{System Overview}

\section{Flow of Data and Procedures}

The ISA control and monitoring system manages numeruus flows of dala and procedures ${ }^{13}$. It is, therefore, reasonable to consider the control of this traffic as the most basic function. As shown in Fig.4, the originals of all data and procedures existing within the system are kept in a global data base (or a set of data bases). One or several working copies of the data and procedures are spread over various parts of the system. They may exist:

(1) In the global data bases;

(2) as working copies interfacing to the Manipulation. Environment ${ }^{*}$

(3) as working coples interfacing to the Machine Environment;

* A control system deals, as a minimum, with three global environments 9 .

\section{Figure 3}

Events in a Malfunction. Detection and Description Process

1. PRECISION (RESOLUTION TIME).

2. AVAILABILITY (RESPONSE TIME). 3. COMPLETENESS (CONTENT)

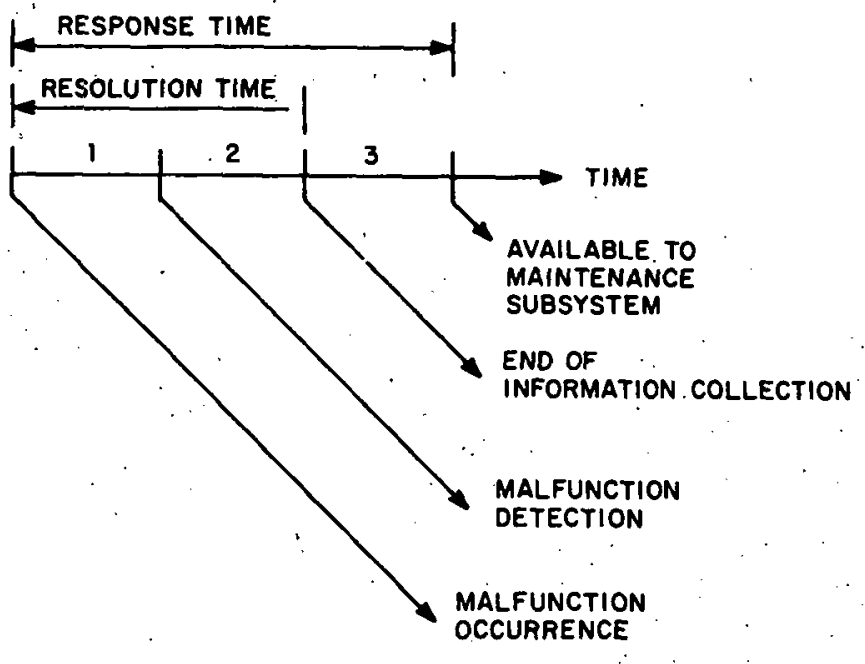


(4) as working copies interfacing to the Automatic $\dot{c}$ Output Environment.

If working copies are being modifled, created or released, then network internal update cycles take care of the appropriate update of the originals.

\section{Figure 4}

Global Data and Control Flow in the ISA Control and Monitoring System.

\section{AUTOMATIC OUTPUT ENVIRONMENT}

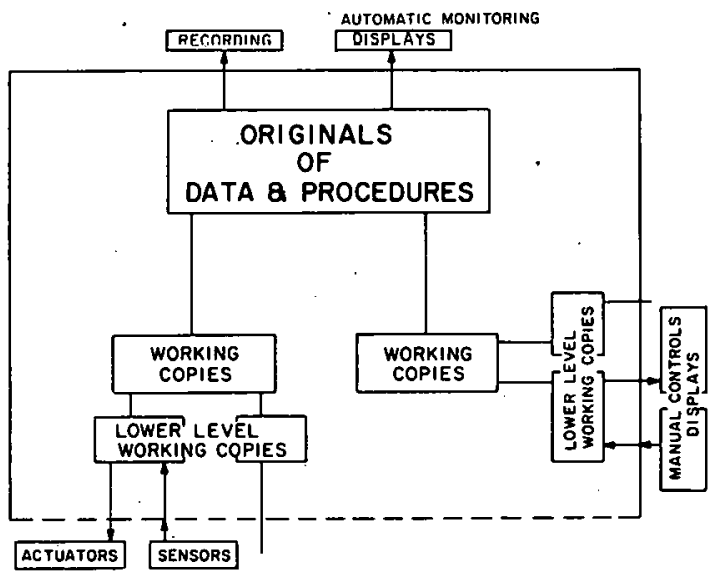

MACHINE ENVIRONMENT

The time interval between the change of a parameter at the source and the updating of the original data base content determines the time resolution of the data base information and, thus, of the system. It is generally true for real-time systems that there is a close relationship between cost on one hand and the required resolution as well as the required modification frequency on the other hand.

Global Architecture. The data and procedure flow

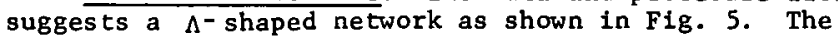
global functional subsystems are listed below.

(1) A set of common nodes contains all service functions which are shared by the local nodes within the network.

(2) A set of local control application* nodes is connected to the common nodes. The application nodes are physically located as closely as economically and otherwise feastble to the appropriate machine subsystems to be controlled. It is likely that, once they are in routine operation, they will be remotely commanded from manipulation nodes as explained subsequently.

(3) A set of local operator manipulation nodes is also connected to the common nodes. They operate the various terminals, graphic monitors, control consoles, etc., in the main control room and in the local control rooms. The manipulation nodes provide the means of communication between operators $* * *$ and the network.
The three sets of nodes are interconnected via a long distance transaction communication subsystem.

\section{Transaction Communication Subsystem}

\section{Communication Lines}

The control application nodes, as well as the manipulation nodes, are connected to the common nodes by $\log 1-$ cal point-to-point long distance communication lines as shown schematically in Fig. 6. In addition, the local nodes are connected to a set of conceptually significant multi-drop lines as shown in Fig. 7 and as described below.

External Event Signals. Functions to be executed in an accelerator are potentially correlated among nodes in time and in accelerator specific events or event sequences. Lines carrying these events are distributed, as needed, throughout the accelerator and are connected directly to the nodes requiring the input. Examples are numerous; for instance, at the acceleration phase a signal will be generated carrying the digitized values of main magnetic field measurements. The resolution time of these signals is significantly influenced by the transfer time in the cables around the rings. It is expected that a resolution time of approximately $20 \mu \mathrm{sec}$ can be safely achieved.

\section{Figure 5 \\ Global Architecture}

\section{AUTOMATIC OUTPUT ENVIRONMENT}

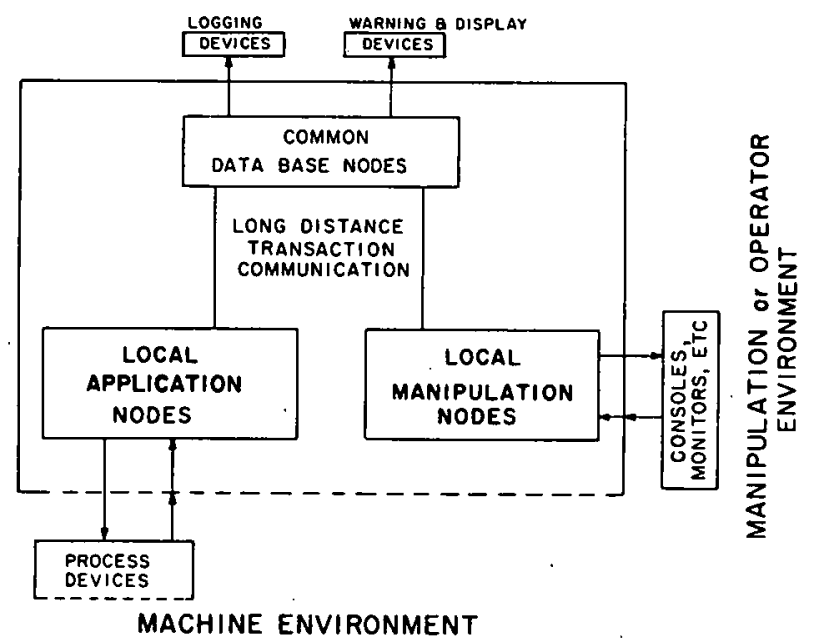

* An application node executes application programs or procedures. The term "application" procedure is used here, as a compromise, to describe the various accelerator control and monitoring procedures which are executed in the network. "Control procedure" and "Control nodes" would probably be more descriptive terms. There is, however, concern about possible confusion resulting from the use of the term control in too many different meanings.

*** The term operator will be used here rather loosely to mean anyone communicating with the machine. The operator may be a machine developer, an equipment engineer, an experimental physicist making adjustments at the intersection regions or, indeed, an operator of the accelerator. 


\section{Figure. 6}

Transaction Communication Subsystem

\section{COMMON NODES}
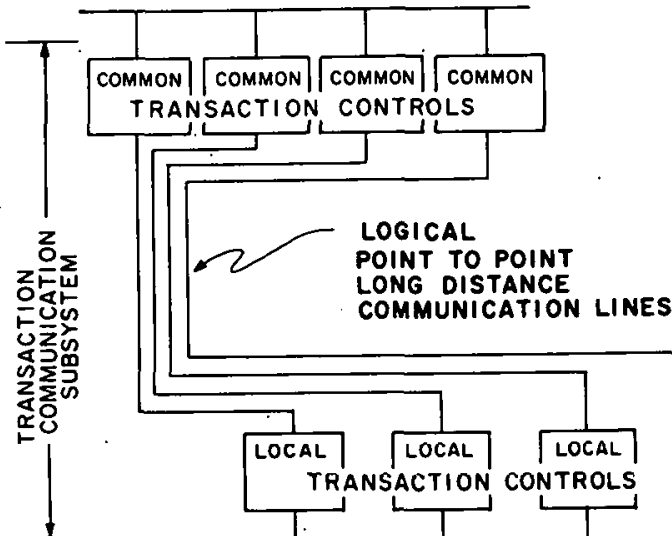

COMMUNICATION LINES

\section{Figure 7}

Connections to a Solicited Transaction Subsystem Terminal.

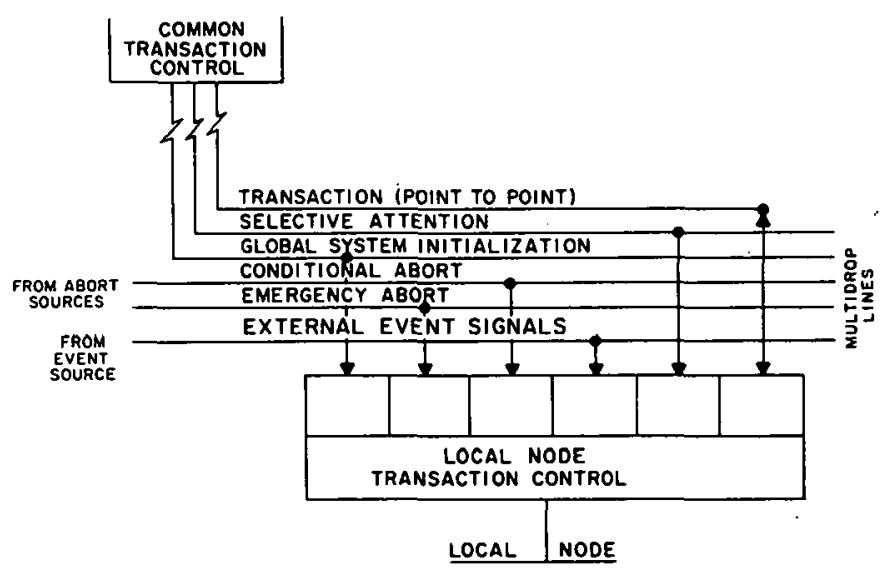

Figure 8

Transaction Protocol

at the Example of a Procedure Transfer

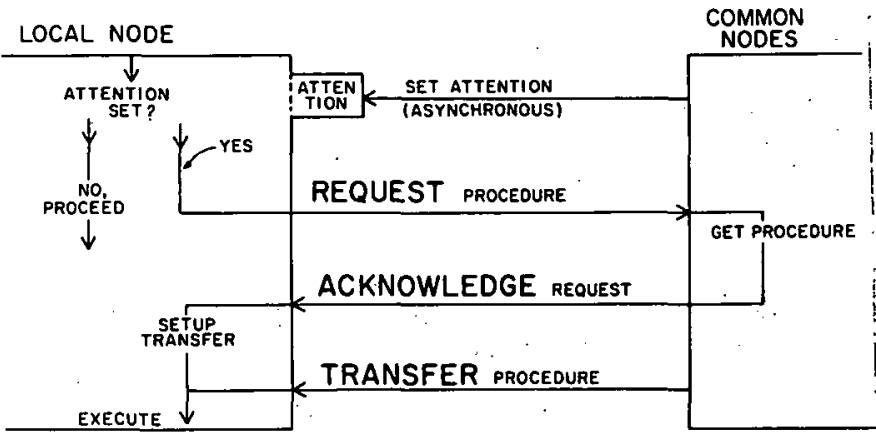

Initialization and Abort Signals. It is a nontrivial task to identify an idle state of the network. The network may be set to this state using the global initialization or abort signals after:

(1) a power turn-on; or

(2) a shutdown of the accelerator caused either by a controlled action or by an emergency action.

\section{Transaction Protocol}

A control application node or a manipulation node requiring or submitting a block of data or a procedure from or to the common nodes initiates a request/response cycle, here called a transaction. A transaction consists of 3 phases as indicated in Fig. 8:

the REQUEST phase;

the ACKNOWLEDGE phase; and

the TRANSFER data or procedure phase.

It should be noted that control application nodes or manipulation nodes always function as requestors, or masters, within the network, and the common nodes always function as responders, or slaves. A request is always initiated by a master. In the case where a control application node is remotely requested to execute a new procedure, an extended transaction protocol upplies. The asynchronous selective attention $\mathrm{flag}$, as shown in Figures 7 and 8 , is raised and is interrogated voluntarily by the approprlate master node. The master node, then, Initiates the above described request/response cycle.

In both the REQUEST and ACKNOWLEDGE phases a block of transaction control information of predetermined length and predetermined structure is transmitted. In the TRANSFER phase, data or procedures as mutually agreed upon in the previous two phases are transmitted. Practical block sizes are $2^{5} \times 16$-bit words for the request and acknowledge blocks and $2^{13} \times 16$-bit words for the transfer block. A reasonable transmission rate of the subsystem would be $2^{17} \times 16-b i t$ words per second.

\section{Reliability and Technology}

The transaction communication subsystem may be considered the skeleton of the network. Malfunction effects proliferating into this subsystem may cause the system as a whole to malfunction. They are, thus, to be avoided. It is necessary that:

(1) at every point of entry to the subsystem an exhaustive test is made as to the format consistency of a transaction to be transmitted. Transaction control and destination address information are part of the format. The subsystem is transparent to the content of a transaction;

(2) the components of the subsystem are of equal or higher reliability than the components in the rest of the network; and

(3) the subsystem itself is structured so that the effects of malfunctions, whether they are caused by hardware deterioration, erroneous operation or software design errors, stay confined to a small part of the transaction communication subsystem.

The latter statement is of particular significance in view of the fact that the communication subsystem is physically distributed along the entire accelerator. Detection and correction of a proliferating malfunction could be a nightmarish and expensive experience. 
This subsystem would be the wrong place for saving of development and construction cost. The subsystem follows logically a point-to-point communication protocol. Serial, unidirectional multi-drop ring structures are often used in present systems because they result in low cable costs. However, there are endless opportunities for proliferation of malfunction effects throughout such a system. The apparent savings in construction costs would likely decrease system reliability and increase subsequent operating cost. Such a structure would not meet above indicated requirements. A presently favored approach is to use a component technology from a well established or rapidly progressing field and to develop a system based un these cumponents.

The components developed for CATV (Community Antenna Television) systems are well established, produced in large quantities and of good quality within reasonable prices. A structurally straightforward system with low malfunction proliferation probability would use one coaxial bidirectional, semi-rigid, broad-band cable per master node - common node interconnection. These cables are mass-produced and cost approximately 60 cents per meter. The transmission protocol can be either bit-serial or word-parallel using time division multiplexing techniques. Several manufacturers are actively engaged in the development of two-way digital and analog transmission techniques based on CATV components.

The technology of optical fiber transmission cables is advancing rapidly. It is vigorously pursued by the telephone companies. In such a technology, which would have to be well established and successfully applied at the time when ISA construction starts, one would probably use one or a few optical fiber lines for each point-to-point interconnection. The optical fiber technology would be preferable because of its insensitivity to electromagnetic noise.

\section{Communication Subsys tem Terminals}

Every master node-to-common node connection includes two terminals: one at the common node and one at the appropriate master node. The master node terminal includes a solicited transaction subsystem, the common node terminal is part of the unsolicited transaction subsystem. Whereas the unsolicited transaction subsystem is an integral part of a subsequently described common node cluster, the solicited transaction subsystem constitutes an Independent functional node as described below.

Solicited Transaction Subsystem. Each solicited transaction subsystem node includes a preprogrammed microprocess or and read-only memory for transaction buffers and for temporary variables. A transaction originates and terminates here. It is, as mentioned earlier, a significant function of the subsystem to provide an effective filter against the effects of externally generated malfunctions.

Figure 9 shows a possible configuration of such a subsystem. A remotely commanded application node is signaled through the prcviously mentioned attention signal via a terminal entry switch. It would be posisible to connect to such a terminal a cluster of application nodes with a terminal entry switch for each individual node.

As presently conceived, the traffic at a given terminal is expected to be sufficiently low so that the cost and complexity of transaction queuing can be avoided. However, this matter is subject to continued study. An intermediate and not very costly step would be to provide separate buffer areas for the attention signal triggered transactions' which have to be kept in storage in the subsystem until the transaction is picked up by the application node.
Figure 9

Possible Configuration of a Solicited Transaction Subsystem Node.

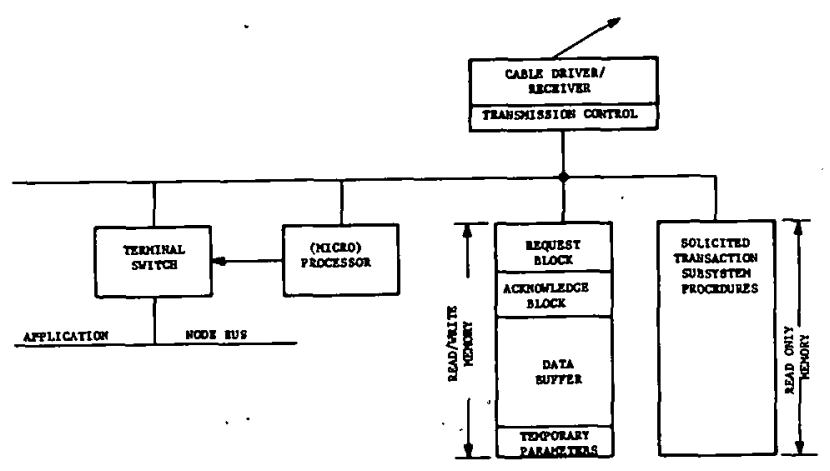

\section{Local Control Application Nodes}

Local control application nodes can be considered either self-sufficient computers or master nodes within the network. Sensors, actuators, instruments, and associated electronic signal conditioning equipment, in the following referred to as process devices, are connected to these nodes. A typical control application node is assembled of several well defined subnodes. The particular configuration, in hardware and in software, of each application node depends on the specific set of function to be performed.

Long distance communication of digital quantities should stay confined to the transaction communication subsystem. It therefore follows that in the assignment of functions to application nodes the general philosophy will prevail to locate a node as close to the process devices as possible. In a presently considered layout, a block diagram of which is shown in Fig. 10, one application node will be located in each of the experiment halls around the accelerator rings. Each one of "these application nodes will service all process devices which are geographically concentrated along one sextant of the accelerator. Thus, those application nodes will be shared among different machine systems, such as the vacuum control system, the magnet correction system, etc. Accelerator systems for which the process devices are concentrated in a confined geographical area will be serviced by functionally distributed application nodes. All functions to be performed for a particular accelerator system will be confined to one of those nodes. The node, then, will be located geographically close to the appropriate accelerator system. Typical systems in this category are the main magnet power supply system, the refrigeration system, the radio frequency system, the injection system and the beam dump system.

\section{Operating Modes} modes:

The application nodes may operate in one of two

(1) the manually commanded" mode; and

(2) the remotely commanded mode.

In the manually commanded mode a local node control device, typically a keyboard/printer terminal, is connected. Commanding of procedures to be executed, such as selection, transportation, Initialization, interruption and termination is conducted manually by a person via the node control device. Conversaiions take

${ }^{*}$ A command is a request which is initiated by an operator from the manipulation environment. A command may cause, in the remotely commanded mode, one or several transactions within the network. 


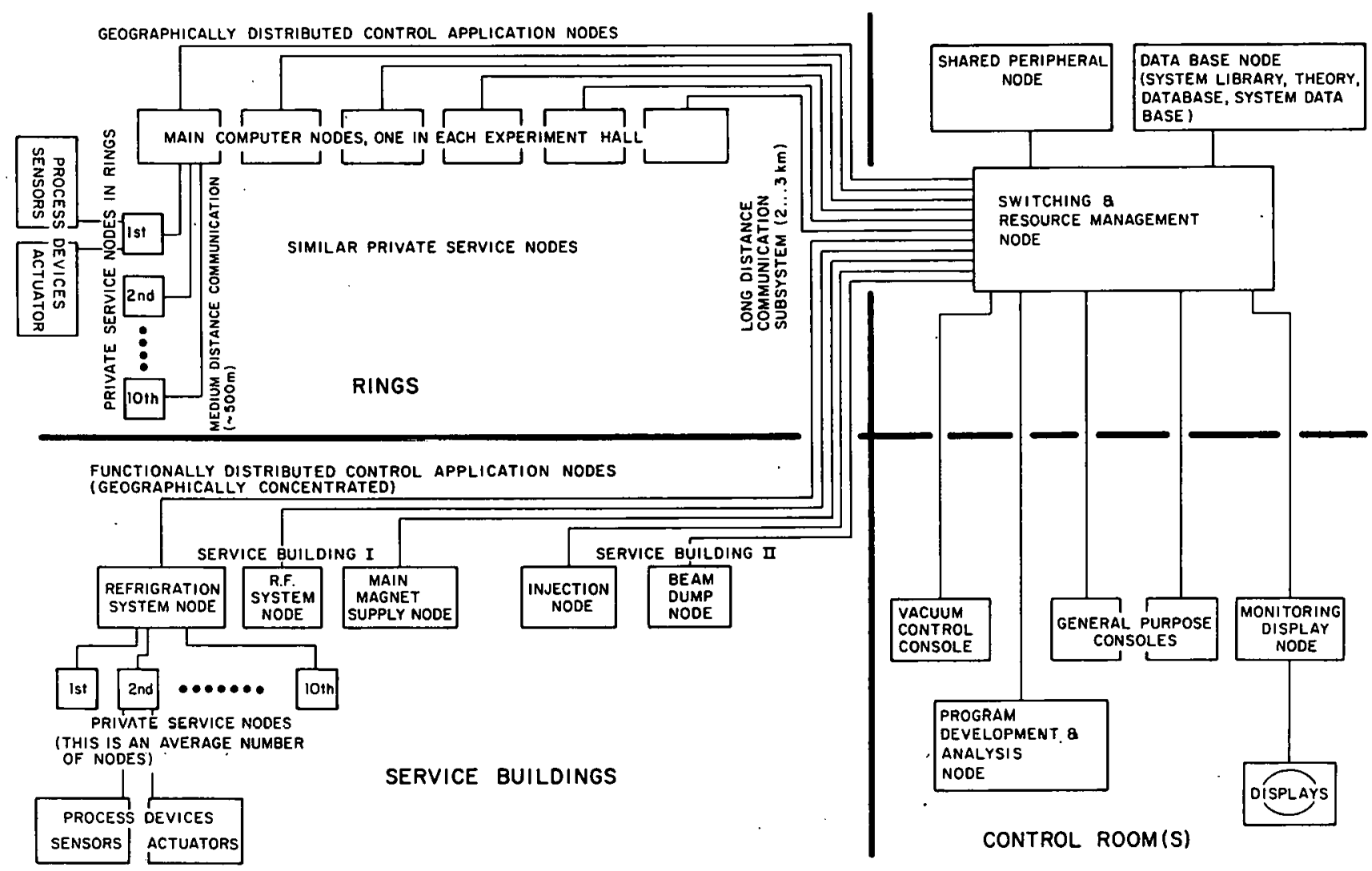

Figure 10

Possible Layout of the ISA Control and Monitoring System

place via a conversational terminal device. The control device can be shared for this function. A conversation is here defined as a sequence of request/ response interactions between a procedure and a person, where the procedure requests information from the person. It is noteworthy that such a control device can be physically remotely located from the node, for instance, the node could be located in the ring structure or in an experiment hall and the control device could be located in the main control room. The manually commanded mode will likely be used extensively during the construction of the accelerator for testing, modifying and operating the various accelerator components and systems.

In the remotely comnanded mode procedures are commanded via the long distance transaction communication subsystem. No node control device is connected. Conversations within a procedure could conceivably take place via a connected terminal.

It is a critical requirement for a control application node that the switch from the menually commanded mode to the remotely commanded mode and vice versa can be done in an uncomplicated fashion which does not require hardware, system software or application software modifications.

\section{Modularity}

It 1s, for obvious reasons, significant for the economy in development costs., subsequent operating $\cos t$, the reliability and the maintainability of the network that the various control application nodes are (1) of modular structure consisting of a small number of different types of modules, and (2) flexible, so that presently known functions can be performed and those to be defined can be accommodated either by existing module types or by incremental addition of new module types. structural modifications should be avoidable during the development phase and in the operational phase 3 of the system. A module may be defined as a subsystem or a nested set of subsystems within the two implementation structures or within the functional architecture. Such a module may represent a substantial portion of the control network. For example, a typical control application node is a module resembling a functional node which likely contains several subnodes.

\section{Configuration}

A possible configuration of an elaborate control application node is shown in Fig. 11. The configuration includes one master node which maintains a master relationship toward the common nodes of the network as well as toward a set of private service nodes. The process devices are interconnected to these private service nodes. The private service nodes receive and execute service requests from the master node. Ușally, several private service nodes exist within such an application node. Communication among the various nodes is accomplished via a shared memory resource to be described later.

\section{Master Node}

Some of the conceptually significant subsystems within the distributed function architecture have been developed and put into routine operation in other control system $14-2 \mathfrak{I}$, over the past several years. In these implementations, processors of the PDP-11 
family* have been used. In order to avoid confusion, the subsequent discussions use the properties of the PDP 11 as the underlying hardware structure, in particular the instruction set, the 16-bit per word organization and the UNIBUS*** Other suitable families of computer processors that are either available now or will be in the future, can, of course, be substituted. As substantiated in the subsequent discussions, the 16-bit per word organization, which is widespread in present minicomputer processors, causes limitations, which hopefully will be alleviated in future processor structures. The limitations are in both the precision of the hardwired arithmetic and in the restricted logical address space. In addition, FORTRAN has been used as the application procedure language. Again, it is possible to substitute another suitable language.

\section{Figure 11}

Configuration of an Elaborate Control Application Node.

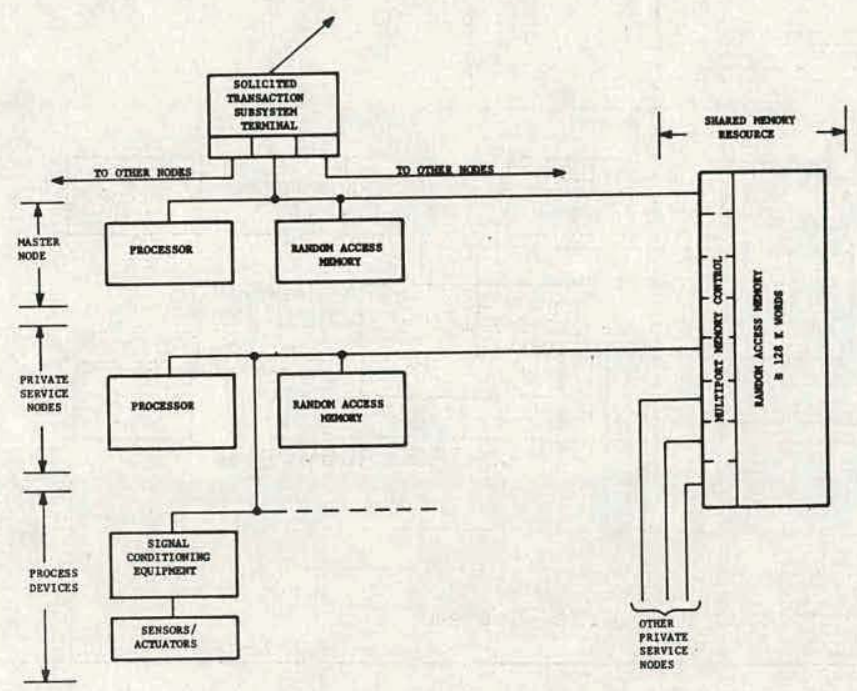

A master node processor is likely a minicomputer processor, such as the PDP11/34, with a memory management provided for two functions:

(1) an extention of the 16-bit word address; and

(2) a protection between the various software subsystems against accidental software destruction.

Typically, two procedures operate at any given time in this node, a standard procedure and a priority procedure differing in their allowable response times. Figure 12 shows a possible assignment of random-access memory space. The size of a load module image of a procedure is $2^{13}$ words for a resident section and $2^{13}$ words for an overlay section 14 . $2^{13}$ words are reserved for local data arrays and parameter arrays. A master node supervisor procedure could have a size of $2^{13}$ words.

\section{Bus Structures}

The various components of the master node as well as those of the subsequently mentioned private service nodes are interconnected by busses. A suitable bus structure has the following conceptual specifications:

(1) minimum restrictions on the node hardware structure and on mechanical measurements of components connected to the bus;
Figure 12

Possible Assignment of Random-Access Memory in the Master Node of a Control Application Node.

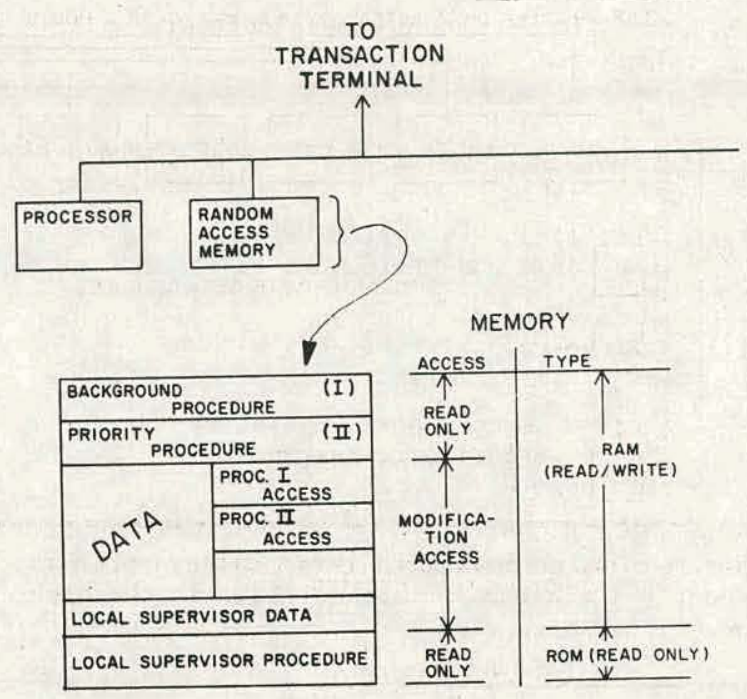

(2) uncommitted data and control flow directions within the bus protocol (master/ slave protocol);

(3) a flexible interconnection architecture for the connection of multiple busses within the nodes and,if needed, to other nodes;

(4) provision for a separation between the data and the control flow; and

(5) an asynchronous request/response relationship of the control and data flows at all levels.

In addition, the bus structure should enjoy widespread acceptance in the industry. The latter requirement is of economic importance. The large quantities of hardware used in such an architecture make it mandatory to rely, to a large extent, on the purchase of reliable, low-cost, mass-produced hardware components such as processors, random-access memory assemblies, interfaces, peripheral controllers, process devices, etc. The UNIBUS satisfies above requirements reasonably satisfactorily. The major disadvantage is its limited physical address space of 218 bytes. The TILINE bus of the Texas Instruments Corporation also seems to satisfy these requirements and does not have the address space restriction. At this time, however, it does not seem to be in widespread use.

\section{Shared Memory Resource Subsystem}

The random-access shared memory resource subsystem, which has been mentioned several times, is discussed in detail elsewhere ${ }^{15}$. Because it is a conceptually significant device it is probably worthwhile to summarize here its functions. A shared memory resource subsystem consists of:

(1) a multi-port memory control which provides access from several processors to the various memory segments of the resource;

*Manufactured by the Digital Equipment Corporation.

${ }^{* *}$ Registered trademark. 
(2) one or several memory segments; and

(3) the allocation and access software which operates at each of the accessing nodes.

Multiport Memory Control. Figure 13 shows a block diagram of the multiport memory control consisting of:
(1) a set of access ports where one functional node is connected to each port;
(2) an internal tri-state bus with a bus arbitrator; and
(3) a set of memory ports where one memory segment is connected to each port.

Figure 14 shows a photograph of an implemtation of a multiport memory oontrol with three access ports and consequently three node busses as shown in the upper portion of the photograph. could be connected to such an access port. A PDP11 processor has a logical address space of $32 \mathrm{~K}$ words. As shown in Fig. 15 a window in the logical address space between the $24 \mathrm{~K}$ word address and the $28 \mathrm{~K}$ word address is used for random access to the physical address space of the resource.

Allocation. The shared memory resource is logically partitioned into allocation units of $2^{12}$ words. For allocation simplicity the following rules apply:

(1) a11 arrays are assumed to be three dimensional;

(2) the linear address is calculated using the following algorithm:

$A=((i-1)+I *(j-1)+I * J *(k-1)) * N$ where

$I=\mathrm{X}$ dimension;

$\mathrm{J}=\mathrm{Y}$ dimension;

$\mathrm{K}=\mathrm{Z}$ dimension;

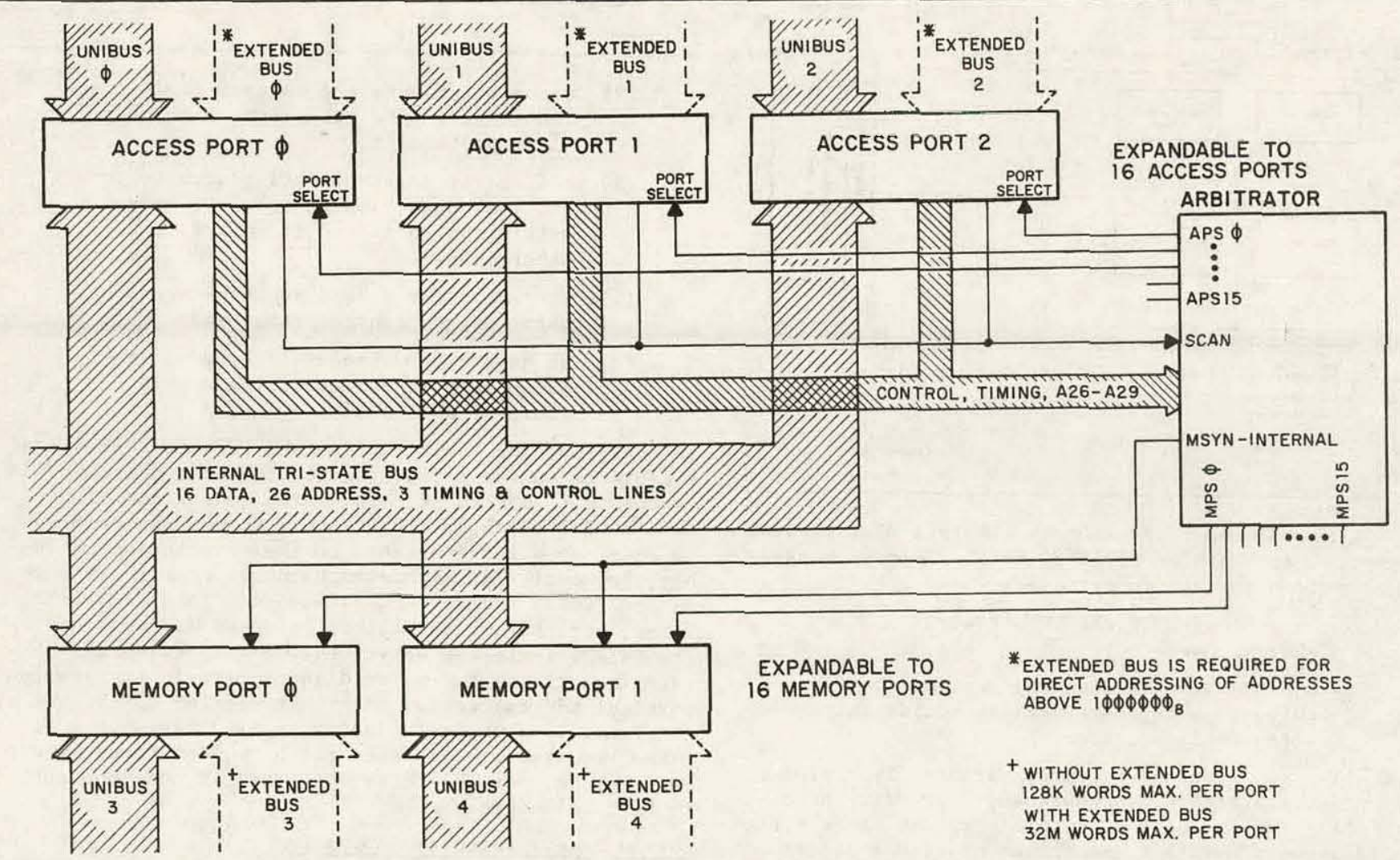

\section{Figure $13 \uparrow$}

Data and Control Flow in a Multiport Memory Control

\section{Figure $14 \rightarrow$}

Implementation of a Multiport Memory Control

Access Ports. In this implemtation, up to 16 functional nodes can be connected to the shared memory resource subsystem via up to 16 access ports. An access port communicates with the connected processor via the node bus. Either a UNIBUS or an extended UNIBUS is used. The address space of the extended UNIBUS is $2^{30}$ bytes using the address lines of a second, parallel connected bus. The PDP11 is one of the processors that

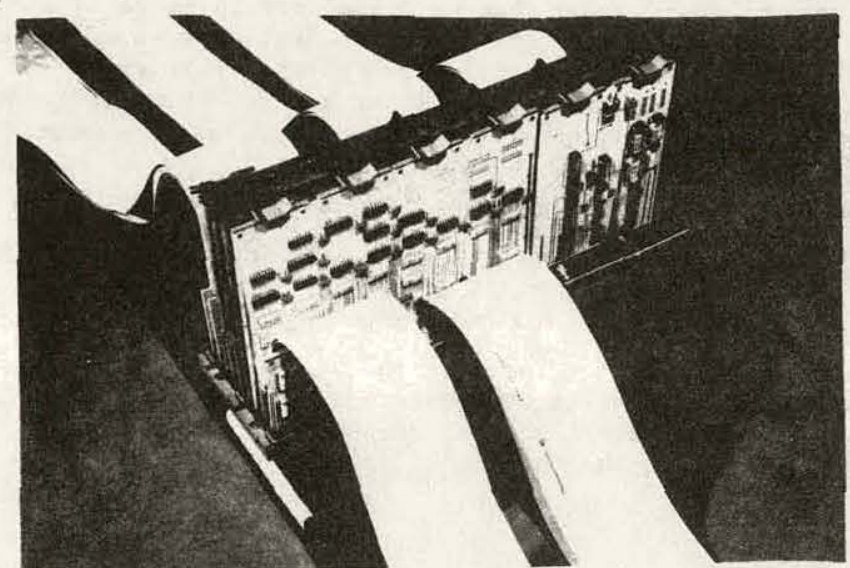


Possible Address Space Allocation for Shared Memory Subsys tem Access

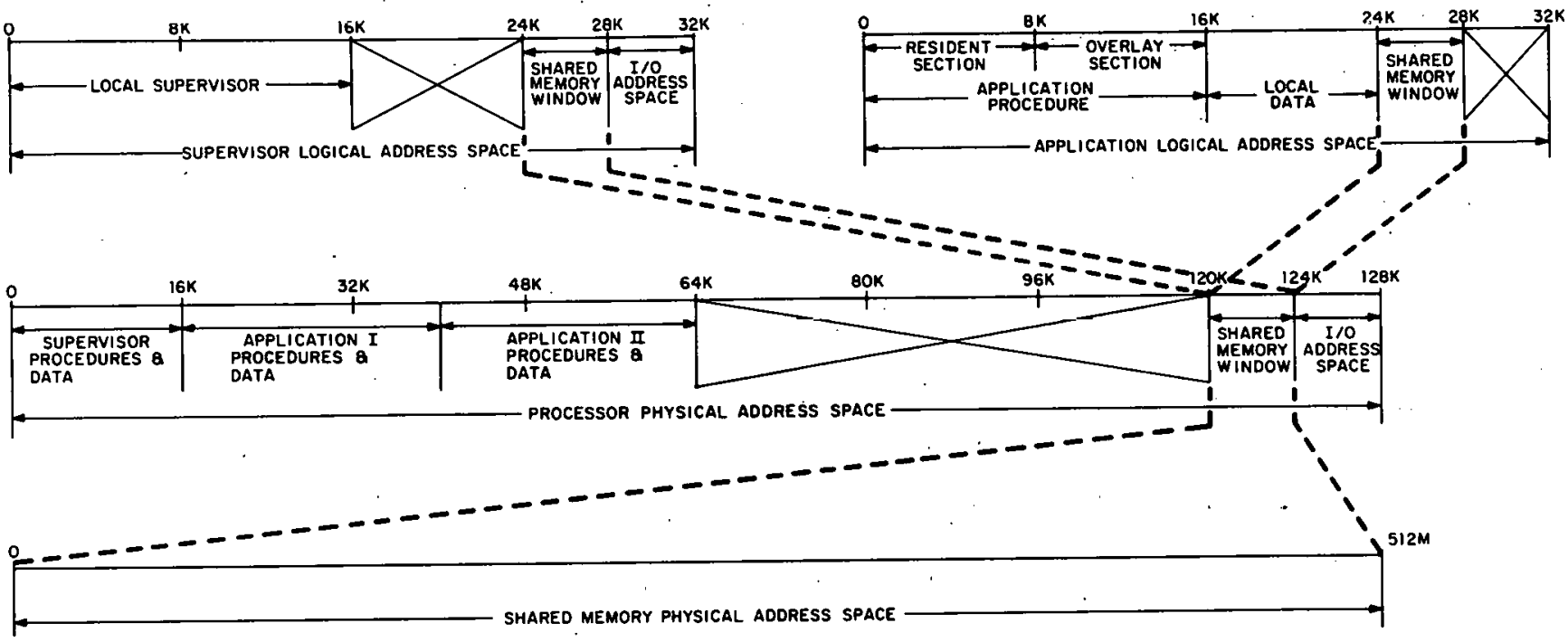

Figure $16 \rightarrow$

Relationships in the Shared Memory Resource Directory

\section{$i, j, k=$ indices for the array ele- ment to be accessed; and \\ $\mathrm{N}$ = data type (byte, word, double word)}

Allocation can only take place by the master node within the application node. The allocation information is kept in a directory including:

(1) a master memory definition table;

(2) a logical array allocation table;

(3) an array allocation directory; and

(4) an array allocation unit map.

Figure 16 shows a block diagram of the relationships of the various components in the directory.

Access and Storage. Access of data array elements from a FORTRAN program is provided by using FUNCTION subprograms. They are, for the various data types:

\begin{tabular}{|c|c|c|}
\hline BYTV $=\operatorname{EMRDBY}($ IARRAY $, \mathrm{I}, \mathrm{J}, \mathrm{K}, \mathrm{IS})$ & for & bytes \\
\hline INTV $=\operatorname{EMRDSI}($ IARRAY $, \mathrm{I}, \mathrm{J}, \mathrm{K}, \mathrm{IS})$ & for & $\begin{array}{l}\text { integer } \\
\text { variables }\end{array}$ \\
\hline INTD $=$ EMRDDI (IARRAY , I $, J, K, I S)$ & for & $\begin{array}{l}\text { double word } \\
\text { integer vart- } \\
\text { ables }\end{array}$ \\
\hline
\end{tabular}

RELV $=$ EMRDSR(IARRAY, I,J, $\mathrm{K}, \mathrm{IS})$ for real variables Storage of data array elements from a FORTRAN program is provided by SUBROUTINEs. They are, for the various data types:

CALL EMWTBY (IARRAY, I, J, K,BYTV, IS) for bytes CALL EMWTSI (IARRAY,I , J, K,INTV,IS) for integer variables

CALL EMWTDI (IARRAY, I, J, K, INTD, IS) for double word integer varibles

CALL EMWTSR(IARRAY,I,J, K,RELV,IS) for real variables.

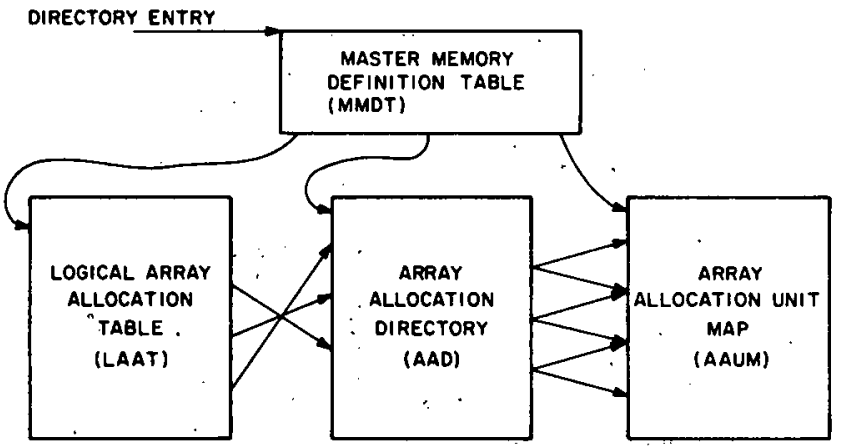

In all of above the variables have the following meanings:

$I, J, K,=$ array subscripts

IS = error return integer.variable

BYTV, INTV, INTD, RELV = varlable to be stored

\section{Private Service Nodes}

A private service node is a functional subsystem which recelves and executes requests from a master node. As mentioned previously the process devices are.connected to such a node. The configuration of a node depends on the number and on the type of process devices as well as on the complexity of functions to be performed by the process devices. It can become very function specific as; for instance, in the case where data is collected from beam monitoring equipment and histograms are generated in real time.

Often such a node operates as a programmed controller to process devices. Its purpose is to execute discrete functions or sequences of discrete functions concerning those process devices on the request of either one of the procedures in the master node. In this case each process device accessible by a private service node is described by a process device descriptor block. The following information is, in part, contained:

(1) device status;

(2) upper and lower limits of device value(s); 
(3) upper and lower alarm limit values*;

(4) calibration constants;

(5) latest measured value(s);

(6) temporary and permanent parameters concerning execution of sequences of discrete functions.

The descriptor blocks are located in the shared memory resource. They are, thus, accessible by the appropriate private service node as well as by the master node:

Communication Protocol. A service request queue for each procedure in the master node is provided in the shared memory resource: The queues operate in a first-in-first-out discipline. The service requests may address discrete functions or sequences of discrete functions. Presently two classes of services seem to be identified:

(1) control action services; and

(2) data acquisition services.

Both service classes may request either:

(1) a single action or measurement in which case a single response is recorded in the descriptor block; or

(2) multiple actions or measurements executed according to a schedule based on one of the external event signals. In this case the management of the responses is complex and has to be determined for the individual functions.:

An example of the latter type of multiple action ts the stepping of the parallel connected magnet correction coils in synchrónization with the main magnetic field according to an individually determined algorithm during the acceleration phase of the ISA. Such an algorithm may require real-time calculations or may require a lookup of prerecorded tables.

Hardware Considerations. A typical private service node will contain a microcomputer processor and random-access memory. For economy and simplicity considerations it is highly advantageous if the instruc-tion set of such a processor and the hardware bus structure were equal to or a subset of the one used in the master node. In the case of the PDPII family the LSIIl microcomputer would be a likely, but not entirely satisfactory candidate. The main objection is the different bus structure of the LSIIl. Special nodes with fixed programs do not, of course, fall under this recommended strategy. The latter may be numerous, for instance, in the case of special data collection processors operating as private service nodes.

Software Considerations. There is no node control device. Therefore, the main program, once initialized, operates in a loop. It is preferably coded in FORTRAN or another suitable high level language. It will pick up service requests from the appropriate queues, initialize the appropriate functions via SUBROUTINE CALLs which are likely coded in a combination of FORTRAN and of a processor specific assembly language. It will test the functions in progress for their status and, if necessary, prepare the response information."

A considerable development effort will be necessary to classify the functions into nested sets each of which include functions of similar purpose, structure and complexity: As an example, subsets of data acquisition functions are:
(1) MEASURE function the current value of a specified process device is obtained and recorded in the process device descriptor block;

(2) MONITOR function

the value of a specified process device is obtained within specified intervals of time or of external event signals;

\section{(3) HISTORY function}

the value of a specified process device is obtained. within specified intervals of time or external event signals. The values are recorded in an appropriate history file in the shared memory resource.

Medium Distance Communication. The distances between a master node location and a private service node location may extend to approximately 500 meters in the geographically distributed control application nodes. There are two classes of communication protocols which may be adopted in the necessary interconnection between a private service node and the shared memory resource.

In the first class the shared memory resource would be physically located at the master node site. The point-to-point connection would logically constitute an extension of the appropriate private service node bus. The advantage is that the random-access feature through the window in the logical address space of the private service node is preserved. Thus, access to the process device descriptor blocks and other shared information could be accomplished in the previously described manner. The disadvantages are the increased access time of the private service node processor to the data in the shared memory resource and probably the high cost.

In the second class of a communication subsystem a high-speed point-to-point connunication line would be used. This approach would require that the process device descriptor blocks and other shared information be located in the local memory of the private service nodes. The master node would access the information via service requests as described before. The advantage is a probably less expensive communication system. Disadvantages result from the elimination of random access to the shared information by the master node.

\section{Process Device Subsystema}

Rapid advances in electronic technology, particularly in microminiaturization, have, as indicated before, their most unsettling impact in the signal conditioning equipment subsystems and the process devices. For instance, a new hybrid device, such as a high-resolution analog-to-digital converter offered by a manufacturer, may possibly upset expensive, long-term development plans. It has been attempted, in the past, to bring this situation somewhat under control by imposing manufacturer independent structural standards on the industry which where based on technologies and

*There are several methods which can be applied for actions on alarm conditions within the distributed function architecture. They depend, in part, on the required response time and on the degree to which geographically distributed information must be correlated. Restrictions in the size of the report do not allow for further discussion of this subject. 
structures existing at the time of the creation of the standard. This however, was the wrong cure because it caused freezing of the technology and subsequent patchwork to cover the failure of the approach.

The distributed function architecturc allows a more flexible view of the situation. Technology decisions concerning the process device subsystems can be made independently of other more stable subsystems in the network, such as data bases, communication between nodes, etc. Also, decisions can be made later when more is known about the accelerator systems to be controlled. In fact, the architecture allows for several different types of process device subsystems. It is, for instance, reasonable to consider a different technology for the low-level analog measurements required in the refrigeration system from the one used in the largely high-level analog and digitally cnntrolled magnet power supply system.

It appears that the correct philosophy to follow is to look, at a time when the requirements are more exactly known, for technologies and devices which (1) follow industry trends, (2) are advanced, and (3) are suitable for the measurement problems being considered. There is evidence that the industry will offer a great variety of devices, structures and technologies in the coming years.

\section{Common Node Cluster}

Common Nodes are those nodes which provide shared services within the network. Shared services may be requested by any control application node or by any manipulation node. It follows that common nodes always operate in a slave mode relationship. Typical shared services to be provided are those concerning:

(1) a data base including all data which are shared among the various nodes within the network;

(2) a pool of peripherals which is to be shared for economic and logistic reasons;

(3) the switching of the transactions from the originating node to the appropriate shared service; and

(4) a network resource management, to be described below.

There exists extensive literature ${ }^{9,16-20}$ describing the purpose and the internal structures of a common node operating system and of various service subsystems. In the case referred to all shared service subsystems are confined to one common node. A summary of the features of the operating system and the subsystems would be beyond the scope of this report. The reader is, therefore, referred to the above references. The ISA control and monitoring system requires substantially extended shared services as well as additional shared services. It is, however, reasonable to use the implemented case referred to as a basis, because the various subsystems have defined boundaries and defined communication protocols. The software subsystems, thus, can be rearranged into an extended hardware structure so that they serve as a basis for a cluster of common nodes. Each node in the cluster will, typically, include a processor, such as a PDP $11 / 45$, $128 \mathrm{~K}$ words of memory, a disk of appropriate size, and if necessary, peripheral equipment. Figure 17 shows a block diagram of a possible configuration of a cluster of common nodes. The nodes are assumed to be physically close to each other.

\section{Cluster Communication}

Communication among the various nodes in the cluster is accomplished using a shared memory resource.
The resource contains the necessary queues and staging areas for transactions to be processed and transactions in progress by the various nodes. A realistic estimate of the size of the random-access memory would be onehalf million words. .

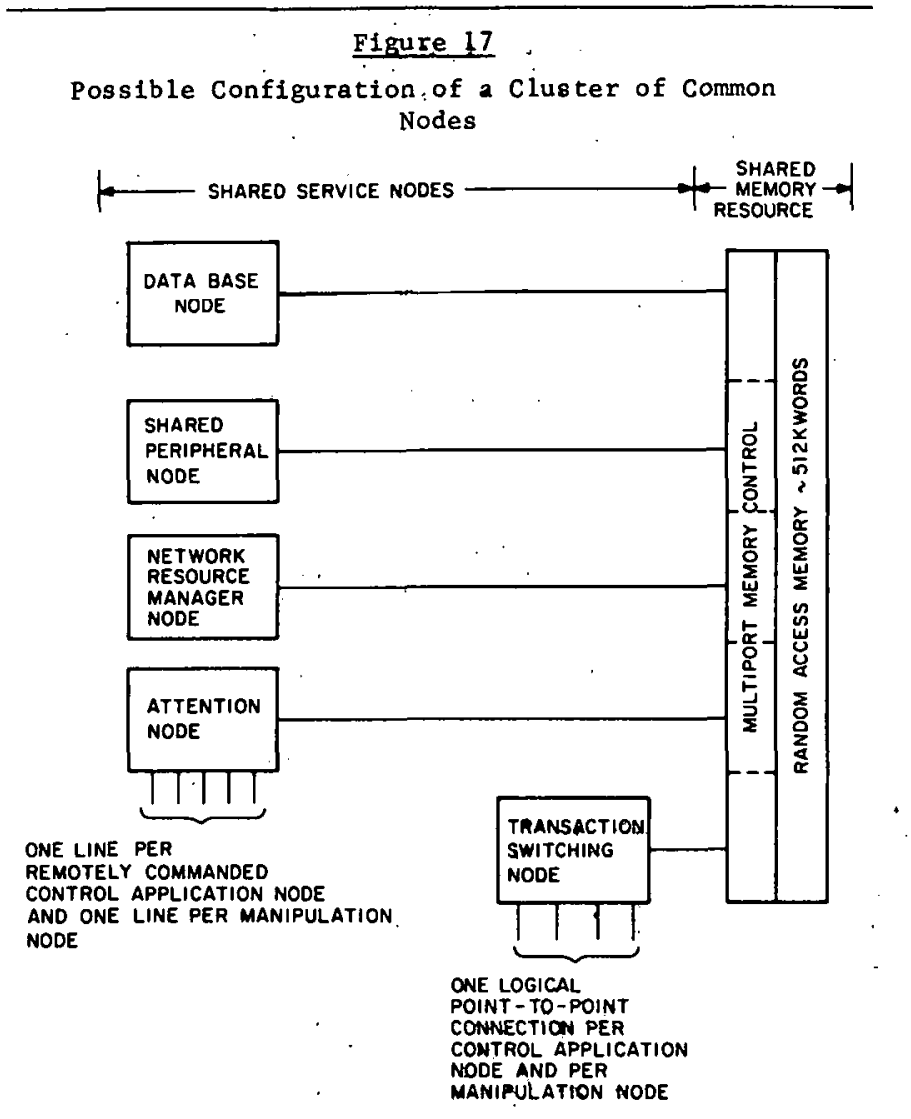

\section{Transaction Switching Node}

The transaction switching node receives transaction request blocks, as described previously, determines the address of the destination shared service node and inserts the request into the appropriate queue. The node also submits acknowledge blocks and, if specified, data blocks to the appropriate originator of a request. The switching node is transparent to the content of a transaction.

Data Base Node

The data base node contains, in an organized form, those data which are" accessed from several master nodes within the network. - Data base contents which are of interest to one node only could be, but are not necessarily stored and managed here. Several requests to the data base may be submitted concurrently from different sources. Therefore, the node subsystem can process several requests simultaneously. Requests which cannot be started immediately are queued in the shared memory resource. In line with the definition of a transaction, each request will cause a response to the originating node by returning an acknowledge block containing, at least, the disposition of the request. The node hardware will include a disk unit of at least 100 million words. Possibly a magnetic tape unit could be included which would be employed as a journal-and data base-dump unit as is customary in data base applications for keeping a back-up record of semi-permanent data base contents. A more detalled description of the operating system structures in such a node is given elsewhere $16,19,19$. 


\section{Shared Peripheral Node}

The shared peripheral node manages requests to output peripherals which are shared in the network for one or several of the following reasons:

(1) they represent a considerable investment, such as a high-speed printer, a high-speed magnetic tape unit; or

(2) the output originates from remote locations and is routed to a centrally accessible place; or

(3) the output medium is logically shared between several control application or manipulation nodes as is the case for history files, accelerator status $\log$, etc.

A detalled description of a possible software subsystem in this node is given olsewhere ${ }^{18}$.

\section{Network Resource Manager Node}

As mentioned earlier, the various control application nodes often execute functions which are correlated with each other. It follows that an operator may originate a command at one of the consoles in the control room which generates transactions to several remotely commanded control application nodce concurrently. The function of partitioning the command into suitable transactions and of merging the responses for the return to the command originator is accomplished by the network resource manager node.

For execution of the command partitioning function, the network resource manager node requires a complete record of all resources and their interconnection paths in the network. In particular, the following records are necessary.

(1) A resource map describes the properties and the correlations of the physical resources in the paths to the remately commanded application node or application node clusters and to the process devices.

(2) Another resource map describes the properties and the correlations of the logical resources contained within above mentioned physical resources. Logical resources may consist of procedures or control parameter blocks. which are permanently located at the application nodes, dynamically as signed buffers along the path to the process devices, etc.

(3) Libraries in the resource manager node contain procedures 14 and control parameters 20 used among the master nodes.

A command originating from a manipulation node is examined as to its resource requirements. The following conditions are, in part, tested:

(1) the availability of the physical resources;

(2) the avallability of the permanently located logical resources; and

(3) the avallability of the required procedures and control parameters in the libraries.

A transaction is released for execution if all necessary resources are claimed. This strategy avoids mutual interlocks of transactions in progress. In a simple case the command requires only resources which are located along a single path. The command transaction which has originated from the manipulation node is then completed and a new transaction is a taged for the appropriate control application node. The attention node, a rather simple microprocessor based node which is connected to the shared memory resource, is instructed to signal the appropriate application node through a onelogical-channel-per-node trigger line. The application node then submits, voluntarily, a request to the resource manager node regarding the staged transaction. A posstble response to the command will be managed in a similar fashion.

A more complex case arises when a command from a manipulation node requires resources which exist along several paths and several control application nodes. The resource manager stages several transactions, one for each path where regources are required. It follows, of course, that a given procedure or control parameter block in the libraries must refer to resources existing along a single path only. The subsystem will then accept the responsibility to merge the information which arrives from the application nodes by independent transactions into a single transaction which then is staged for the command originating manipulation node.

\section{Redundancy}

If the requirement arises that the reliability of the communication network must be increased by the use of redundancy, then several expansions and reconfigurations without modifications can be made during development and after deployment of the network. For instance, a configuration of multiple paths between the operator environment and the control application nodes would be configured by distributing the transaction switching subsystem to redundant nodes using redundant physical paths. The introduction of a redundant network resource manager node would be, for logistic reasons, difficult. Here, improved reliability and protection against loss of resource map information can be, if necessary, achieved by using recording techniques which are well established in the data base technology, such as employing an independent node which inserts Into a history file a record of every modification in the resource maps associated with periodic selective or global dumps of the resource maps. In the case of a failure, the information can be reconstructed starting from the last dump. It is, however, subject to further study to determine whether those extreme reliability measures would be justifled for the ISA control and monitoring system.

\section{Manipulation Nodes}

Manipulation nodes are used, as indicated previously where operators interact with the network. In an accelerator such as the ISA, the following mantpulation nodes would be needed as a minimum.

(1) Global control consoles allow operator access to all machine systems.

(2) Local control consoles allow access to specific machine systems. For example, such a console is probably desirable for the vacuum system of the accelerator. The process devices are distributed along the rings and are serviced by several geographically distributed control application nodes. The access of the vacuum console manipulation node would be restricted to these process devices by using access protection codes in the network resource manager subsystem. 
(3) Large numbers of display monitors will probably be distributed in the control room, the service buildings and in the experimental halls. These monitors are managed by one or several display manipulation nodes.

(4) A conventional data processing system is necessary as another manipulation node.

(5) Provision must be made for very convenient development of application procedures (programs) to be used in the network.

\section{Application Procedure Development}

Application procedure (program) development is the process of creating, correcting, assembling, converting, testing, confining and filing executable modules of procedures. Generally it is an effort by a person to successively integrate information which is available in a highly dispersed form and in a variety of styles. The effort is, for obvious reasons, (1) very susceptible to human errors and (2) highly iterative. An effective organization of such a process is disciplined, defined in considerable detail and automated along the information flow starting from the information input by a person and ending at the properly filed executable module. Numerous tests and filters should, ideally, be inoorporated along the information flow in order to minimize the penetration of human error effects into the executable module: It is quite remarkable that the computer science field did, up to now, not seem to succeed in defining this process adequately and in determining methods for convenient automation. Not infrequently, justifications for fundamental system architecture parameters and properties of the implementation $s$ tructures are based on their supposed advantages to procedure development convenience. A detailed discussion of this issue would be beyond the scope of this report. In the following, a few observations are given.

As shown in Fig. 18, a typical procedure development process is partitioned into three subprocesses. The text editing process deals with the creation and the iterative correction of source language modules and with the insertion of those modules into a source module library. The process is quite similar to the one necessary for compiling a letter or a report. The word processing industry pursues automation vigorously. A variety of suitable techniques and devices will probably be developed there in the coming years which are usable for convenient automation of the text editing process. Flexible disks seem to have gained acceptance as a medium for the storage of text and, thus, of source module libraries.

In the compilation process a source module is converted into an object module which contains code on the level of a computer processor instruction set. The module is then inserted into an object module library. In the linking process a series of object module librarles are searched and required object modules are copied and linked (bound) into an executable load module. The load module, then, is inserted into a procedure library. The linking process has the tendency to become exceedingly complex.

Whereas the editing process can be conveniently confined in a microprocessor based programmable terminal as they become increasingly avallable, the compiling and linking process requires a medium sized computer node with at least a disk unit and a high-speed printer. As described alsewhere 14 it is possible and desirable to automate these two processes to a high degree of convenience so that a person developing a procedure is. unconcerned about the internal complexities of these
Figure 18

Procedure Development Processes

INPUT

PROCESS

कUTPUT
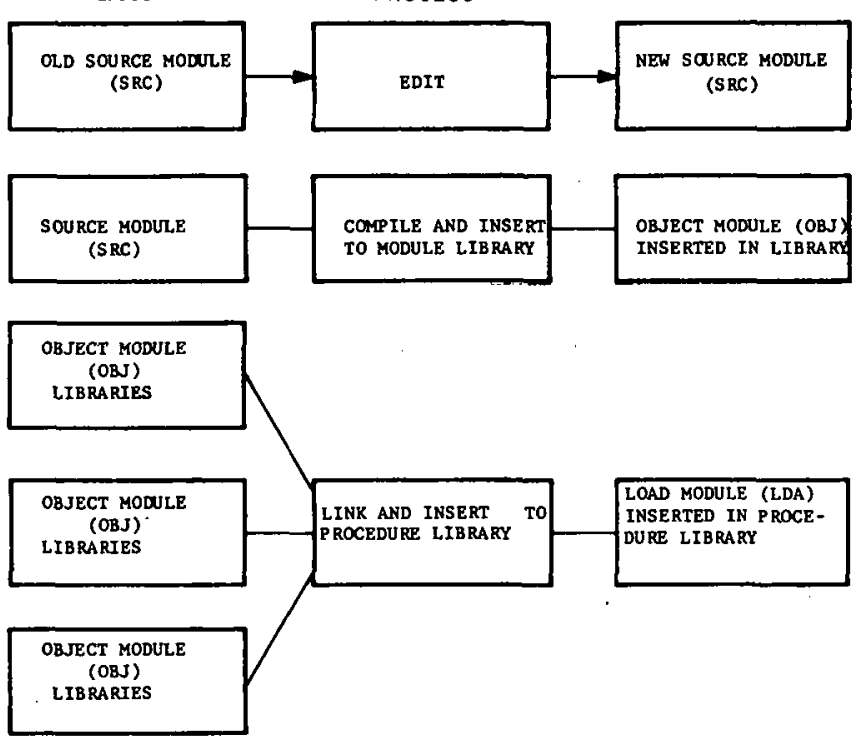

processes as they arise in library managements, labelling conventions and in the sequencing of the various portions of the processes.

Source modules can also be directly executed by the use of interpreters. Programs coded in the BASIC language are almost exclusively executed interpretively. This method, which is very successfully used in small systems such as programmable desk calculators and small, self-contained data acquisition systems, has its problems In a system of the complexity as described here. In the interpretive method one source module statement at a time is implicitly compiled, here better described as interpreted, and linked concurrently with the execution process. The most apparent disadvantage is in the lack of tests and filters between the information input by a person and the executable module. There is also a peralty in increased execution time because the implicit compilation and linking processes have to be repeated at every execution of a statement. Particularly the linking process can become prohibitively slow in procedures which contain many nested source modules. Justifications brought forward are conveniences gained by the elimination of explicit compilation and linking and system simplifications gained by the elimination of structural differences between data and procedures. Both justifications, however, appear to have their basis in the fallure of the computer science field to determine methods for adequate automation of the development of application procedures in the first place.

\section{Future Studies}

In addition to further studies on various portions of the system architecture increased emphasis will be given to studies concerning the numerous specific control requirements of the various accelerator systems. In this effort descriptions of the system architecture will serve as an underlying structure. Further construction and testing of conceptually significant system components are planned. 


\section{Acknowledgements}

The author would like to thank Dr. M. Q. Barton, Dr. H. Hahn and Dr. J. R. Sanford for their support of this study, and Dr. V. Radeka for encouragement and valuable advice.

It is a pleasure to acknowledge many contributions by the members of the Systems Group in the Instrumentation Division. Special appreciation is extended to Dr. F. W. Stubblefield for significant contributions to the subject of common node concepts, Dr. W. H. Hardy, II, for designing and constructing a shared memory resource subsystem and to Mr. S. Rankowitz for extensive discussions concerning process devices and signal conditioning technology. Also, the programing asisistance of Mr. N. E. Greenlaw and Mrs. M. A. Kelley is appreciated.

Acknowledgement is given to Mr. R. Frankel, Dr. $J$. W. Humphrey and other members of the ISA Division of the Accelerator Department for useful discussions.

\section{References}

1. M. C. Crowley-Milling. The Design of the Control System for the SPS. CERN 75-20, December 1975.

2. A Proposal for a Positron-Electron CollidingBeam. Storage Ring Project (PEP). LBL Rep. 2688 , SLAC Rep. 171; April 1974.

3. K. B. Mallory. Control Through a System of Small Computers. IEEE Trans. Nuc1. Sci., NS-22, 3, June 1975.

4. R. E. Daniels, R. W. Goodwin and M. R. Storm. The NAL Computer Control System. IEEE Trans. Nucl. Sci., NS-20, 3, June 1973.

5. ISABELLE, A Proposal for Construction of a ProtonProton Storage Accelerator Facility, BNL 50519, May 1976.

6. C. G. Bell, A. Newell. Computer Structures: Readings and Examples, p. 11, McGraw-Hill, 1971.

7. Ibid, P. 3.

8. R. Keyser, The Use of Computers for Accelerator Control. National Conference on Particle Accelerators, Moscow, 1972.

9. D. G. Dimmler. Functional Distribution - An Architecture for Multi-User Computer Networks in Instrumentation. IEEE Trans. Nuc1. Sci., NS-21, 1, February 1974.

10. D. G. Dimmler. Study for the Expansion of the OnLine Data Processing Facility. BNL 50180 ( $\mathrm{T}-536)$, June 1968.

11. F. Beck. The Design and Construction of a Control Centre for the CERN SPS Accelerator. CERN SPS$\mathrm{Co} / 76-1$.

12. D. G. Dimmler. Architecture of a Maintenance Subsystem in a Multi-User Computer Installation. Proc. Workshop on Fault Detection and Diagnosis in Digital Circuits and Systems. Allentown, Pennsylvania, December 1970.

13. D. G. Dimmler. The ISABELLE Control and Monitoring System - Global Overview. CRISP 75-5, April 1975.

14. D. G. Dimmler, N. Greenlaw, M. A. Kelley, D. W. Potter, S. Rankowitz and F. W. Stubblefield. The Brookhaven Reactor Experiment Control Facility A Distributed Function Computer Network. IEEE Trans. Nuc1. Sci., NS-23, 1, February 1976.
15. D. G. Dimmler and W. H. Hardy, II. A Shared Random Access Memory Resource for Multiprocessor Real-Time Systems. IEEE Trans. Nucl. Sci., NS-24, 1, February 1977.

16. F. W. Stubblefield and D. G. Dimmler. Transaction Processing in the Common Node of a Distributed Function Laboratory Computer System. IEEE Trans. Nuc1. Sci., NS-22, 1, February 1975.

17. F. W. Stubblefield and D. G. Dimmler. A Task Scheduler and Service Subsystem for the Common Node of a Distributed Function Laboratory Computer Network. IEEE Trans. Nucl. Sci., NS-23, 1 , February 1976.

18. F. W. Stubblefield. Continuous Sharing of a Record-Oriented Output Device Within a Distributed Function Laboratory Computer Network. IEEE Trans, Nucl. Sci., NS-23, 1, February-1976.

19. F. W. Stubblefield. Logical and Physical Resource Management in the Common Node of a Distributed Function Laboratory Computer Network. IEEE Trans. Nuc1. Sci., NS-23, 1, February 1976.

20. F. W. Stubblefield. A File Management of Experiment Control Parameters Within a Distributed Function Computer Network. IEEE Irans. Nucl, Sci., NS-24, 1, February 1977.

21. D. G. Dimmler, N. Greenlaw, and S. Rankowitz. A Controllable Automated Environmental Data Acquisition and Monitoring System. IEEE Trans. Nucl. Sci., NS-23, 1, February 1976 